\title{
La Educación Intercultural Bilingüe: Representaciones y Prácticas Sociales de la Otredad
}

\author{
The Intercultural Bilingual Education: \\ Representations and Social Practices of the \\ Otherness
}

\section{L'Éducation Interculturelle Bilingue : Representations et Pratiques Sociales de l'Autre}

\author{
Francisca de la Maza \\ Pontificia Universidad Católica de Chile
}

RESUMEN

Se presenta una discusión teórica y metodológica para el análisis de programas de Educación Intercultural Bilingüe (EIB) en Chile. Se propone un enfoque que profundiza en la aplicación de políticas nacionales a los ámbitos locales, destacando el rol que asume el agente estatal local en su realización y en la construcción del Estado y, en particular, en su relación con los pueblos indígenas. A partir del análisis de este programa se profundiza en las representaciones y prácticas sociales que se transmiten respecto a la otredad indígena a partir del discurso de los profesores.

Descriptores: política social, representaciones sociales, educación intercultural bilingue.

\section{ABSTRACT}

It is presented an argumentative theoretical and methodological discussion for the analysis of intercultural bilingual education programs (EIB) in Chile. It is proposed an approach that deepens into the implementation of national policies to local areas, highlighting the role assumed by the local government agent in its performance and in the construction of the state and, particularly in its relationship with the indigenous peoples. Based on the analysis of this program, it is deepened in the representations and social practices that are transmitted in respect to the indigenous otherness from the teachers' speech.

Key words: social policy, social representations, bilingual intercultural education. 


\section{RÉSUMÉ}

Une discussion théorique et méthodologique pour l'analyse des programmes d'Education Interculturelle Bilingue (EIB) du Chili est en cours de mise en place. Il est proposé de réaliser une étude qui se centrera sur l'application des politiques nationales au niveau local, en soulignant le rôle qu'assume l'agent de l'Etat dans leur réalisation et dans l'amélioration des relations entre l'Etat et les populations indigènes. Cette analyse permettra d'en savoir plus sur les représentations et pratiques sociales de l'autre indigène qui sont transmises aux élèves via le discours des professeurs.

Mots-clés : politique sociale, représentations sociales, éducation interculturelle bilingue.

\section{Introducción}

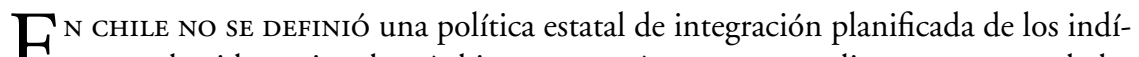
- genas a la vida nacional, más bien se generó por otros medios como parte de las políticas nacionales de desarrollo y distribución del territorio, desde fines del siglo XIX, cuando finalizó el proceso de ocupación del territorio mapuche por parte del ejército chileno. Sin embargo, esto no significa que se ignoró al indígena, sino que se utilizaron otros mecanismos para la construcción del Estado -nación y su proyecto nacional unitario, teniendo mucha importancia instituciones como la iglesia y las escuelas.

A partir de 1990, en Chile con el retorno a la democracia, se inicia una nueva relación entre el Estado chileno y pueblos indígenas con un compromiso gubernamental de crear mecanismos políticos e institucionales para llevar a cabo una política estatal y una legislación dirigida a los pueblos indígenas. Con la aprobación de la ley 19.253 y la creación de la Corporación Nacional de Desarrollo Indígena (CONADI) como organismo regulador de la política indígena comienza una nueva etapa en la historia de la relación Estado chileno y pueblos indígenas.

Por este motivo, la política social hacia indígenas generada a partir de los 90' se puede considerar como un cambio paradigmático en la historia del Estado chileno. Sin embargo, la ley, la creación de una institucionalidad diferenciada y el desarrollo de programas sociales específicos para indígenas, no fueron suficientes para cambiar la relación entre sociedad indígena y no indígena y, menos aún, entre Estado y pueblos indígenas. El asunto es más complejo y va más allá de políticas. Desde la perspectiva de este análisis, la transformación de la relación debe basarse en un impacto en las representaciones sociales que se manifiestan en prácticas y acciones concretas entre Estado y pueblos indígenas.

En este sentido, se presenta un análisis de la aplicación de un programa de educación dirigida a indígenas para profundizar en las representaciones y prácticas de la otredad, particularmente vinculadas a la educación intercultural bilingüe. Esto en un contexto nacional de visibilización de los indígenas y desde la perspectiva de la etnografía del Estado, las representaciones y prácticas sociales. Se postula que estos aspectos son claves para la construcción de la otredad indígena en el imaginario nacional.

\section{La EIB como Práctica de Política Social: Enfoque y Método}

El Programa de Educación Intercultural Bilingüe (EIB) impulsado por el Ministerio 
de Educación (MINEDUC) y más tarde en conjunto con el Programa Orígenes ${ }^{1}$ se inicia dentro del marco de la nueva política de los pueblos indígenas de los gobiernos de la Concertación ${ }^{2}$, definidos a partir de acuerdos políticos con organizaciones indígenas, respaldados y exigidos dentro de un contexto de mayor apertura mundial a la temática indígena. Una de las demandas indígenas de diferentes organizaciones es la necesidad de un cambio en los sistemas educativos, debido a que la escuela se considera como una de las fuentes donde más directamente se ha intentado "desindianizar" al indígena y castellanizarlo, en desmedro de la lengua y cultura indígena. Los Programas de EIB son una respuesta a esta demanda.

En Chile la experiencia se inicia con la creación del Programa de EIB del MINEDUC en 1995 en cumplimiento del mandato estipulado en la ley indígena, primero con experiencias pilotos y más tarde con acciones sistemáticas. Diversos estudios $^{3}$ han mostrado que los programas de EIB que se han implementado en las escuelas rurales han enfrentado diferentes dificultades en su aplicación. Esto debido a múltiples factores donde destacan algunos como: falta de competencias pedagógicas de los profesores en su formación inicial y práctica profesional para desarrollar contenidos de interculturalidad y bilingüismo; y relaciones problemáticas entre escuela y comunidad al enfrentar las propuestas de EIB. Algunas investigaciones han privilegiado el estudio del aula por medio de microetnografías para identificar las principales dificultades de estos programas. Por otro lado, desde el MINEDUC se ha fomentado perfeccionamientos de profesores en EIB en ámbitos nacionales y extranjeros.

A partir de este contexto, se plantea que el programa de EIB constituye una política social y permite aportar en el análisis de la perspectiva de la "construcción del Estado desde lo local.” Estos programas están enfocados generalmente para la población con mayores niveles de pobreza como son algunos sectores de comunidades indígenas, sectores con necesidad de "protección social." Este enfoque apunta a analizar las representaciones y prácticas sociales que están detrás de una política social, que se transmiten y materializan en la sociedad por medio de las acciones e interacciones entre los agentes estatales y los involucrados en la política.

Desde esta perspectiva, se postula que las experiencias de EIB son un espacio privilegiado de estudio para analizar la construcción de la otredad dentro del Estado, que se transmite por medio de discursos y prácticas estatales que refuerzan o intentan transformar las representaciones sociales arraigadas en la sociedad. Es un espacio privilegiado porque la EIB no sólo considera un beneficiario indígena, como se hace en general con la política social hacia indígenas a partir de los 90', sino que también contiene una transformación en la política en sí misma, pues contiene cambios curriculares específicos que no contempla una política más global como la que se plantea en ámbitos productivos o sociales.

La concepción del Estado, en la cual se basa esta perspectiva, se relaciona a un concepto (Abrams, 1977), una idea que se construye a través de la práctica estatal y el quehacer de sus instituciones o agencias y de los funcionarios o agentes. Esto significa entender el Estado como una construcción, un proceso ideológico e institucional dinámico, donde tiene importancia la relación local entre agentes estatales y la sociedad en general, pero donde también operan poderes estructurales, especialmente los de 
orden económico y político que superan los ámbitos locales y nacionales.

El análisis de la práctica estatal no significa olvidar que detrás de las políticas sociales está presente la idea de proyecto ideológico y de construcción cultural que define formas aceptables de comportamiento, que repercuten en las representaciones y prácticas sociales de la sociedad. Al contrario, las políticas en sí mismas y los programas sociales realizan clasificaciones sociales e instauran en la identidad colectiva la idea de una sociedad (Corrigan y Sayer, 1985). Lo que se intenta es buscar un ámbito de investigación y análisis que permita ver críticamente la construcción del Estado y la reproducción de formas de actuar en la sociedad.

La concepción del Estado como idea privilegia las prácticas estatales concretas por medio de estudios específicos dentro de las llamadas "etnografías del Estado" (Gupta, $1995)^{4}$ o las "formas cotidianas de la formación del Estado" (Joseph y Nugent, 2002). Esta perspectiva enfoca en el cómo se gobierna y cómo ha sido históricamente la forma de gobernar. Se sugiere superar el enfoque que estudia el Estado desde la óptica de la administración pública o la burocracia pública, sino que se quiere ahondar desde un enfoque sociocultural en la práctica estatal, cobrando relevancia las prácticas de las agencias y agentes y las formas de relación con los miembros de la sociedad o en específico con los "beneficiarios" de dichas políticas.

$\mathrm{Al}$ enfatizar en las representaciones sociales se quiere dar cuenta de la forma como se explica la realidad de los diferentes miembros de ella. Alude al concepto definido por Moscovici (1979), que las entiende como formas cognitivas propias del sentido común o del pensamiento ingenuo, por medio del cual se conoce el mundo y se orienta la acción. Las representaciones son sociales pero se expresan a través del individuo y se construyen a partir de la interacción del individuo con el grupo social. Por medio de las representaciones se puede establecer un espacio de saber común en la sociedad, que tiene el poder de anticipar, prescribir y programar el comportamiento de los individuos. No sólo guían el comportamiento, sino que también remodelan y reconstituyen los elementos del medio en el que el comportamiento debe tener lugar.

Los discursos estatales pueden entenderse como representaciones sociales de la sociedad que moldean el comportamiento y más aún reproducen prácticas, ideas del otro y del uno. Los discursos del Estado se construyen sobre un marco discursivo común que intenta dejar de lado los espacios que puede haber controversias y luchas. Los agentes estatales, al igual que otros miembros de la sociedad, comparten estas representaciones y son los que deben aplicar una política definida desde el nivel central.

Las prácticas cotidianas del Estado forman parte de este proceso cultural y social y se manifiesta a través de formas y rutinas de relación entre agente estatal y sociedad en general y también es generador de identidades sociales. Para esto cobra importancia la forma en que se elaboran las actividades del Estado, sus rutinas y rituales para la constitución y regulación de las identidades sociales. A través de estos agentes y agencias se reproducen las representaciones sociales del Estado y de la otredad y se intenta construir la identidad nacional, fortalecer la idea de sociedad homogénea y unitaria.

En el sector de educación, como en otros sectores, las agencias estatales tienen una jerarquía que van desde un nivel mayor -lugar donde se define dicha política- que 
corresponde al Ministerio de Educación y que por mecanismos institucionalizados se expande a niveles más bajos como las municipalidades y más concretamente en la escuela. En esta trama de jerarquías y niveles estatales se relacionan diversos sujetos sociales que interactúan según las reglas establecidas y quizás más comúnmente según prácticas y rutinas no escritas. Pero también se van reproduciendo representaciones del Estado y en el caso de los programas de EIB se reproducen relaciones y representaciones de la otredad, del indígena y del no indígena.

En este sentido la educación y particularmente la escuela rural o urbana tiene un papel importante en los procesos identitarios de sus alumnos (niños y niñas) que se complementan con otros espacios cotidianos como la familia y la comunidad. Los sistemas educativos como parte de la política estatal van determinando ciertas pautas de comportamiento, valores, relaciones dentro de la sociedad, que constituyen y regulan las identidades sociales.

La EIB enfocada como política social hace referencia a esta construcción del Estado desde abajo, es decir, le da relevancia a la bajada del discurso global, del discurso político a la práctica estatal. Los programas de EIB se consideran como parte de la política nacional, insertos también en tramas globales de política educativa, con una repercusión regional y local. En este caso, son los profesores, las escuelas y sus sostenedores quienes son lo que en algún sentido se convierten en agentes estatales, los que aplican políticas educacionales definidas por el Ministerio de Educación, sean de origen particular o público. Por este motivo, el estudio de la EIB en escuelas visto como política social puede dar elementos que contribuyan a identificar factores que influyen en la construcción de la sociedad chilena, del proyecto nacional, de las representaciones sociales del Estado y sus repercusiones en la práctica del agente y agencia local.

Siguiendo esta línea, el informe de educación de OECD (Organización para la Cooperación y el Desarrollo Económico) señala respecto a los programas de EIB que en ninguna parte es más evidente el rol que juegan los valores más amplios de la sociedad y las instituciones que median la implementación de la política educacional que en las políticas dirigidas a mejorar las oportunidades de los estudiantes indígenas. En consecuencia, en ninguna parte es mayor la brecha entre la retórica y la implementación de política. 5 Sin embargo, no hay que desconocer los logros que se han obtenido con las experiencias pedagógicas durante estos años. Para lo cual es necesario analizarlas e identificar los elementos que se han desarrollado y que han obtenidos logros importantes en diferentes ámbitos.

Junto a esto, el desarrollo y aplicación de los programas de EIB también son un espacio donde manifestaciones de racismo, desconocimiento, indiferencia y clasificaciones pueden presentarse en forma más evidente, como lo demuestran algunos estudios realizados en el aula, ${ }^{6}$ donde se siguen reproduciendo las estructuras de dominación entre sociedad indígena y no indígena. Estos estudios también analizan las ideas sobre la sociedad indígena, en las cuales destacan ideas primordialistas de la cultura indígena, con una tendencia a idealizar el pasado, a mostrar lo "intercultural" y lo "bilingüe" como algo separado de la sociedad no indígena. Esto ayuda a incrementar la idea del indígena como representación social del pasado y no contemporáneo y 
muchas veces como "sociedades simples"; es decir, sin analizarla desde una perspectiva de sociedad compleja donde las relaciones sociales, la construcción de conocimiento y en general cualquier elemento de la cultura indígena tiene múltiples relaciones con la sociedad no indígena.

A partir de este análisis de la escuela como agencia y del profesor como agente es necesario hacer las conexiones y mirada del Estado hacia arriba, es decir, desde la escuela enfocar la política educativa en sus diversos niveles, analizar las redes de información, las propuestas de perfeccionamientos, las formas de financiamiento, los conceptos que se manejan en este nuevo paradigma y cómo lo procesa el agente mismo, cómo se transmite en la sala de clase y cómo lo vive la comunidad en que está inserta la escuela.

Esta perspectiva no pretende juzgar al profesor o a la escuela, sino lo que interesa es analizar y "descubrir" cuáles son las estructuras más profundas - como diría Bourdieu - que llevan a la construcción de representaciones sociales y de procesos identitarios que reproducen y moldean formas de dominación, de discriminación y que probablemente inciden en que los programas de EIB no tengan los éxitos esperados. Aunque también no hay que olvidar que a veces las decisiones políticas y los recursos no son suficientes. Los programas como EIB que plantean una nueva relación Estado y pueblos indígenas, significan un cambio estructural en la política estatal que los gobiernos no siempre están dispuestos a hacer de manera profunda.

La escuela se entiende como una organización abierta afectada por condiciones internas y externas, un espacio más de construcción de Estado y ciudadanía, espacio donde se aplica una política social, lo cual no es algo exclusivo del agente, sino también tiene relación con la población a quien va dirigida dicha política, los beneficiarios y cómo se construyen mecanismos de negociación, acuerdo y conflicto.

\section{La EIB como Experiencia en Dos Escuelas en el sur de Chile}

En el año 2005-2006, se realizó una investigación que abarcó la política social para indígenas en una comuna del sur de Chile, en la Región de los Ríos ${ }^{7}$ (de la Maza, 2007). Se estudiaron varias líneas de investigación que iban desde la escuela, programas sociales generales, programas sociales para indígenas hasta el funcionamiento del municipio. Se realizó una etnografía del Estado desde lo local, enfatizando las interacciones entre los agentes estatales locales y los beneficiarios, líderes y organizaciones mapuches. Esto se efectuó principalmente por medio de observaciones prolongadas a oficinas estatales y acompañamiento al terreno de visitas de funcionarios, participación de eventos públicos, entrevistas a diversos a agentes claves estatales y no estatales y revisión de documentos.

Dentro de esta investigación, se abordó la temática educativa como uno de los ejes centrales de construcción del Estado y de la otredad como representación social, particularmente se analizaron los discursos y prácticas de los agentes estatales que tenían relación con EIB. A continuación se presentan la discusión de algunos resultados relativos a la política educativa de la comuna, particularmente, en dos escuelas pioneras en EIB a nivel nacional. Su objetivo es abrir nuevas preguntas y líneas de debate 
frente a las implicaciones de la política social diferenciada en las representaciones y prácticas sociales.

En la comuna de estudio, se estima que un $10 \%$ del profesorado que trabaja en escuelas rurales es de origen mapuche. Sin embargo, en la Corporación Municipal de Educación, de quien dependen las escuelas municipales, no existe ningún profesional a cargo del tema intercultural, a pesar que hay cinco escuelas que trabajan en el área de EIB, y sólo existe una relación netamente administrativa con esas escuelas. Por lo cual, los programas de EIB son externos a la municipalidad, no forman parte del sistema municipal. Esto tiene relación con la forma que se implementan estos programas, llegando directamente a las escuelas. Esto tiene diversas consecuencias en la relevancia política que puede tener un programa, pues su impacto es sólo en la escuela pero no el sistema educativo comunal general.

Por otro lado, la implementación de programas de EIB se vincula a otros programas dirigidos a la población indígenas, vista como una fuente de recursos adicionales para la escuela:

"Nosotros estamos en la etapa de abrir la cobertura y ver si se puede acceder, porque tenemos dos escuelas más que quieren incorporarse definitivamente al sistema, ellos me han dicho ojalá al tema Orígenes por el tema de la comunidad y las platas que llegan ahí." 8

Según la percepción del director municipal de educación las escuelas tienen interés de incorporarse a la EIB porque pueden obtener "botines", más recursos con programas específicos y no tanto por su importancia educativa.

En las dos escuelas analizadas, la instalación de la EIB fue en 1995 y 1996, en ambas escuelas la iniciativa surge de profesores que no son mapuches y fruto de un interés personal. Estos programas de EIB se adecuaron a los currículos incorporando preferentemente asignaturas de la cultura mapuche, pero no se trabajó la interculturalidad y el bilingüismo. Mientras la cultura mapuche, se aborda desde una perspectiva "objetiva" como un conjunto de rasgos, conceptos, pero no desde una perspectiva dinámica y compleja. Muchos agentes que llevan a la práctica la EIB desconocen aspectos fundamentales de contenidos curriculares que incorporan la interculturalidad y la cultura indígena:

"A nivel de gente que están en cargos "de", a veces hay gente que no tiene idea de la cultura mapuche ocupando un cargo que tiene que ver con lo intercultural, entonces la pertinencia de tener un profesional que conozca el tema, que esté comprometido con eso, es crucial." 9

Por otra parte, respecto a los rendimientos académicos de estos programas, en general las escuelas no obtienen buenos resultados en las pruebas nacionales de medición educacional. La explicación que el director educacional le atribuye es reveladora al traslucir su concepción de lo intercultural: a veces por incentivar lo "intercultural" no se trabajan los aspectos educativos nacionales. Pero por otro lado, si uno analiza el 
contexto de estas escuelas, están insertas en una situación de pobreza:

"Estamos por un lado recuperando la lingüística mapuche, un poco la cultura, pero nosotros tenemos que lograr poner nuestros indicadores que logramos, es decir, todo aquello, nuestros valores wingkas ${ }^{10}$ que aunque no les gusten, son valores universalmente aceptados, entonces ahí entramos con un dolor tremendo, en esos lados es por el tema de la subcultura, aunque no quisiera llamarla así, sino que hay un trabajo muy fuerte que hacer con los apoderados." 11

Frente a este discurso, se puede constatar que se reproduce el discurso del paternalismo frente al otro indígena, una concepción de inferioridad que emerge de las autoridades municipales, donde uno de los responsables según este funcionario es la familia indígena. No hay una lectura estructural de la situación de pobreza de las comunidades rurales mapuches y su impacto en la educación.

Según los relatos de los profesores, la incorporación de la EIB en las mallas curriculares es un intento de contextualizar las salas de clases entregando materiales visuales pertinentes a la cultura mapuche, pero no significa un proceso de acompañamiento de transformación de representaciones y prácticas sociales respecto a la otredad indígena.

Un aspecto fundamental, para comprender la instalación de la EIB en una escuela rural, como los casos estudiados, es la relación comunidad-escuela. En las experiencias de EIB, las familias no siempre están motivadas para implementar programas donde se fomente el uso del mapuzungun ${ }^{12}$ o elementos culturales, incluso en algunas la postura ha sido considerar que no es el rol de la escuela tomar esa iniciativa, muchas veces cuestionando a los monitores o asesores culturales de la comunidad que participan en dichos proyectos. Por otro lado, en la comuna de estudio, muchas de las comunidades son hablantes de mapuzungun, sin embargo por decisión de los mayores se está produciendo un corte generacional en la transmisión de la lengua.

Uno de los aspectos interesantes identificados en el análisis de los discursos de los profesores es que no perciben la diferencia entre pérdida de la lengua y la identidad étnica. Más bien perciben que la identidad se construye por aspectos objetivos como la lengua, la apariencia y el apellido, sin considerar otros aspectos subjetivos relacionados a las interacciones sociales. Como reiteran los profesores que trabajan en EIB, el impacto de estos programas es principalmente en la autoestima de los niños: "se identifican con su raza y se sienten mapuches" porque antes "los niños no se hacían valorar." La directora de una de las escuelas, luego de 10 años de estar incorporada al programa de EIB, señala:

“esta gente es mestiza y como ya están mezclados tienen más rasgos chilenos que mapuche (...) Es una comunidad indígena por el apellido solamente, porque la verdad es que no tienen identidad, porque no tienen longko ${ }^{13}$, ellos no celebran ngillatunes. "14

En el mismo sentido, una de estas escuelas para mostrar el aporte de la EIB presenta un aula llena de artesanía elaborada por los alumnos. Mientras en la otra escuela, se 
celebra el wetripantü (el año nuevo mapuche que corresponde al solsticio de invierno entre el 20 y 24 de junio según el ciclo lunar), con diversas actividades como bailes, comidas, etc., elementos objetivos de la cultura mapuche "tradicional."

Junto a lo anterior, el paternalismo y los indicadores raciales se evidencian en los discursos de los profesores, reproduciendo representaciones o imaginarios "del mapuche" como cerrado y "celoso" de su conocimiento. En este análisis, no consideran las condiciones económicas y políticas que están detrás, sino que mantienen y reproducen el prejuicio, la discriminación e incluso racismo frente al otro:

"Lo que yo percibo de acá es que el profesor mapuche se encierra mucho en su tema, en cambio el profesor wingka o con mezcla en su sangre, creo que tiene esta otra visión de integrar y de la globalización y yo creo que va a buscar muchas más estrategias para lograr eso, porque yo en las conversaciones técnicas que he tenido con la asesora cultural, ella es muy mañosa (mal genio) y dice las cosas muy fuertes y claras, y hay cosas que molestan (...), que es dolor que tiene el pueblo mapuche respecto al pueblo wingka." 15

Reforzando esta idea de racismo, entendido como una construcción social que define a otro como inferior dentro de un contexto particular histórico y social, se puede señalar que está arraigado en la sociedad, como se señala en la siguiente cita del director municipal de educación:

"Yo no he tenido resistencia en los profesores del área rural, el hecho de estar trabajando con estos niños les provoca todo este cambio de rechazo, porque logra conocerlos, porque logra ver que son personitas tan hermosas como otros, como un blanco o un rubio y yo creo que más que nada por la forma en cómo el profesor convive con sus alumnos, yo creo por ahí, el alumno va a aprender cuando sea tratado con cariño." 16

Junto a estos planteamientos, también se refuerza esta idea de agente estatal, profesoralumno, pensado en una interacción también arraigada en la sociedad de una relación unidireccional o verticalista, incluso paternalista, con la comunidad, muchas veces autoritaria. Que en sí mismos, ésta relación e interacción profesor-escuela o profesorcomunidad, es opuesta a la idea de interculturalidad, como un espacio de diálogo que apunta a relaciones en condiciones más igualitarias. Los profesores no mapuches se sienten capaces de desarrollar la EIB, por lo cual estudian por su cuenta la cultura y lengua mapuche, repitiéndose la actitud autoritaria y paternalista de las políticas sociales.

Complementario a esto, la escuela como institución estatal se mantiene al margen de los conflictos políticos de las comunidades y particulares (presentes en una de las escuelas estudiadas), las fronteras étnicas se refuerzan y no se politizan, la escuela se mantiene como un espacio "neutral."

Referente a esto, se puede postular que el rol del profesor chileno o "wingka" como representación social no encaja con la idea de interculturalidad. Como señalan los 
testimonios analizados, tienen enormes dificultades para relacionarse con profesores mapuches, revelando los problemas para comprender y convivir con el otro, reflejando las representaciones de la otredad: los no mapuches se sienten discriminados por los mapuches:

\begin{abstract}
"Nosotros que no somos mapuches siempre andamos en primera fila rescatando estas cosas de interés para que los chicos logren su identidad, pero hay mapuches que no tienen interés alguno, al contrario siempre andan poniendo trabas. Son egoístas con su conocimiento y no lo quieren dar." ${ }^{17}$
\end{abstract}

Las fronteras étnicas se refuerzan por ambos lados. Las representaciones se reproducen y se concretan en prácticas que no reflejan un camino hacia la interculturalidad.

\title{
A Modo de Conclusión
}

En términos generales se puede decir que a los programas de EIB aún están en una etapa de sensibilización respecto a la otredad indígena, considerando como centro la incorporación en el curriculum escolar de elementos de la cultura indígena en términos objetivos (vestimenta, lengua, ceremonias, artesanía, etc.) pero que ha avanzado muy poco respecto a la interculturalidad y el bilingüismo. Hay un conflicto entre lo que es la enseñanza de modos tradicionales mapuches y lo que es realmente "interculturalidad", es decir, un trabajo con metodologías específicas que incorporen diversas culturas, en forma dinámica, con el fin de promover su valorización pero de la convivencia entre culturas. Este tipo de competencias no poseen los profesores, en la mayoría de los casos pues no se incorpora en la malla curricular de formación de profesores, salvo algunos intentos en algunas universidades y en programas de formación de profesores en EIB. La recuperación y/o desarrollo del mapuzungun no es prioridad en la EIB, sino más bien se privilegian contenidos culturales, muchas veces definidos por profesores sin mayor especialidad.

También se puede decir que para el desarrollo de programas de EIB dependen en gran parte del voluntarismo de los profesores, de sus motivaciones e intereses personales el desarrollo de EIB, más que de las comunidades indígenas y de una política educativa que intente transformar una relación de dominación. Por otro lado, intelectuales y líderes indígenas que promueven la EIB se mantienen en un nivel político de reivindicación étnica, pero no desarrollan el aspecto técnico. Más bien promueven un discurso pro-mapuche y de culpabilización al otro wingka con una idealización de lo mapuche, sin una negociación real con los gobiernos nacional y locales. A falta de una propuesta técnica y pedagógica significativa, las experiencias de EIB siguen siendo sólo experiencias, sin un impacto real en la política pública y en la nueva relación Estado-pueblos indígenas. Sin embargo, es necesario destacar que en los últimos años algunos intelectuales indígenas, especialmente profesionales que se han perfeccionado en diversos países, han ido concretando propuestas técnicas para promover la revitalización lingüística del mapuzungun y que promueven la interculturalidad. 
Con el desarrollo de políticas indígenas como la EIB, las estructuras de dominación y representaciones de la otredad indígena se mantienen y aún más se evidencia en forma clara en el actuar de los funcionarios locales. Esto lleva a que finalmente, las prácticas cotidianas de la política social hacia indígenas reproducen los discursos y estructuras de poder ligadas a las representaciones sociales del Estado chileno, por lo tanto, de las identidades sociales chilenas y del otro indígena. Esto corresponde a paradigmas profundos en las estructuras que siguen teniendo un lugar preponderante en la reproducción de representaciones sociales y prácticas gubernamentales, a pesar de los perfeccionamientos, a pesar de las sensibilidades personales y de los discursos del "Nuevo Trato Estado chileno-pueblos indígenas."

\section{Notas}

1. Programa Orígenes es un programa que pretende el desarrollo con identidad, desde una perspectiva sostenible y con pertinencia cultural. Se ejecuta en convenio entre el gobierno de Chile y el BID. Se inició su primera fase el 2001 y la segunda el 2006.

2. Concertación por la Democracia: es una coalición política organizada en torno al retorno de la democracia en el plebiscito de 1988. Convoca a diversos partidos políticos opositores a la dictadura militar.

3. Por ejemplo: PUC (2004), MINEDUC (2005) y Cañulef (2002).

4. Gupta entiende este concepto como el análisis de las prácticas diarias de las burocracias sociales y la construcción de un discurso del Estado en la cultura pública.

5. En OEDC (2004: 281).

6. Como los estudios de PUC (2004) y MINEDUC (2005).

7. Esta región limita al sur con la Región de la Araucanía, que forman gran parte del territorio tradicional mapuche antes de la ocupación militar chilena finalizada en 1883.

8. Entrevista a Director Municipal de Corporación Educacional (de la Maza, 2007).

9. Entrevista a profesora mapuche que participó en los proyectos pilotos (de la Maza, 2007).

10. "Wingka" en mapuzungun se refiere al no mapuche, al chileno, generalmente en forma peyorativa

11. Entrevista a Director Municipal de Corporación Educacional (de la Maza, 2007).

12. "Mapuzungun": Lengua mapuche.

13. Longko: Autoridad mapuche.

14. Nguillatun: ceremonia religiosa mapuche.

15. Entrevista a profesores de escuela con EIB (de la Maza, 2007).

16. Entrevista a profesores de escuela con EIB (de la Maza, 2007).

17. Entrevista a profesores de escuela con EIB (de la Maza, 2007). 


\section{Bibliografía}

Abrams, Philip (1988). "Notes on the difficulty of studying the State (1977)", Journal of Historical Sociology, 1(1) March (pp. 58-89).

Bourdieu, Pierre (2003). La dominación masculina. Barcelona: Anagrama.

Cañulef, Eliseo (2002). Hacia la interculturalidad y el bilingüismo en la educación chilena. Chile, CONADI, FEDER.

Cañulef, Eliseo; Fernández, Emilio et al (2002). Aspectos generales de la educación intercultural bilingüe (EIB) y sus fundamentos. Chile, MINEDUC.

Corrigan, P., Sayer, D., and Aylmer, G.E. (1985). "Introduction", en The Great Arch: English State Formation as Cultural Revolution. Oxford: Basil Blackwell, (pp. 1-13).

Chiodi Francesco y Bahamondes, Miguel (2001). Una escuela, diferentes culturas. Chile, CONADI.

De la Maza, Francisca (2007). Politica social para indígenas: Un análisis desde la perspectiva de la construcción del Estado y la interacción local, Panguipulli (1990-2005), Chile. Tesis para optar al grado de doctora en antropología social. México, Centro de Investigaciones y Estudios Superiores en Antropología Social CIESAS.

Fleury, Sonia (2002). "Políticas sociales y ciudadanía", en Umbrales, núm. 11. La Paz, Bolivia, CIDEAS-UMSA, (pp.189-218).

Gupta, Akhil (1995). "Blurred boundaries: the discourse of corruption, the cultura of politics, and the imagined state", en American Ethnologist 22(2), (pp. 375-402).

Joseph, Gilbert y Nugent Daniel compiladores (2002). Aspectos cotidianos de la formación del Estado. México: Ediciones Era.

Kuper, Adam (2003). "The return of the native", en Current Anthropology 44(3) June (pp.389-402).

MINEDUC-Programa de EIB (2005), "Prácticas pedagógicas que favorecen la Educación Intercultural Bilingüe”, elaborado por Pontificia Universidad Católica de Chile, Sede Villarrica, Chile, Colección Estudios en EIB.

Moscovici, Sergue (1979). El psicoanálisis su imagen y su público. Argentina, Colección temas básicos, Editorial Huemul, cap. 1-3, (pp. 5-89).

OECD- Organización para la Cooperación y el Desarrollo Económico (2004). Revisión de politicas nacionales de educación Chile. Chile, Publicaciones MINEDUC.

Opazo, Marlene, Huentecura, Jorge (2002). Algunas orientaciones curriculares para le educación intercultural bilingüe en contextos mapuches. Chile, MINEDUC.

PUC-Pontificia Universidad Católica Sede Villarrica (2004). "Estudio Etnográfico realizado en escuelas rurales de las Regiones IX y X", Proyecto Discriminación y Pluralismo Cultural en la escuela. Chile, UNESCO, 2004.

Saldívar, Emiko (2002). Everyday practices of indigenismo: an ethnography of Mexico's Instituto Nacional Indigenista. Submitted to the Graduate Faculty of Political and Social Science of the New School University in partial fulfullment of the requirements for the degree of Doctor of Philosophy, USA.

Stavenhagen, Rodolfo (2001). La cuestión étnica. México, El Colegio de México. 